\title{
LINEAR AND \\ NONLINEAR \\ MHD WAVE PROCESSES \\ IN PLASMAS \\ Final Report
}

PI: John A. Tataronis

Contract No. DE FG02 - 97ER54398 


\section{DISCLAIMER}

This report was prepared as an account of work sponsored by an agency of the United States Government. Neither the United States Government nor any agency Thereof, nor any of their employees, makes any warranty, express or implied, or assumes any legal liability or responsibility for the accuracy, completeness, or usefulness of any information, apparatus, product, or process disclosed, or represents that its use would not infringe privately owned rights. Reference herein to any specific commercial product, process, or service by trade name, trademark, manufacturer, or otherwise does not necessarily constitute or imply its endorsement, recommendation, or favoring by the United States Government or any agency thereof. The views and opinions of authors expressed herein do not necessarily state or reflect those of the United States Government or any agency thereof. 


\section{DISCLAIMER}

Portions of this document may be illegible in electronic image products. Images are produced from the best available original document. 


\title{
LINEAR AND NONLINEAR MHD WAVE PROCESSES IN PLASMAS
}

\author{
PI: John A. Tataronis
}

\begin{abstract}
This program treats theoretically low frequency linear and nonlinear wave processes in magnetized plasmas. A primary objective has been to evaluate the effectiveness of MHD waves to heat plasma and drive current in toroidal configurations. The research covers the following topics: (1) the existence and properties of the MHD continua in plasma equilibria without spatial symmetry; (2) low frequency nonresonant current drive and nonlinear Alfvén wave effects; and (3) nonlinear electron acceleration by $\mathrm{rf}$ and random plasma waves. Results have contributed to the fundamental knowledge base of MHD activity in symmetric and asymmetric toroidal plasmas. Among the accomplishments of this research effort, the following are highlighted:
\end{abstract}

- Identification of the MHD continuum mode singularities in toroidal geometry.

- Derivation of a third order ordinary differential equation that governs nonlinear current drive in the singular layers of the Alfvén continuum modes in axisymmetric toroidal geometry. Bounded solutions of this ODE implies a net average current parallel to the toroidal equilibrium magnetic field.

- Discovery of a new unstable continuum of the linearized MHD equation in axially periodic circular plasma cylinders with shear and incompressibility. This continuum, which we named "accumulation continuum" and which is related to ballooning modes, arises as discrete unstable eigenfrequency accumulate on the imaginary frequency axis in the limit of large mode numbers.

- Development of techniques to control nonlinear electron acceleration through the action of multiple coherent and random plasmas waves.

Two important elements of this program are student participation and student training in plasma theory. 


\title{
LINEAR AND NONLINEAR MHD WAVE PROCESSES IN PLASMAS
}

\author{
PI: John A. Tataronis
}

\section{Summary}

This program advances the theory of low frequency linear and nonlinear wave processes in magnetized plasmas. A primary objective has been to evaluate the effectiveness of MHD waves to heat plasma and drive current in toroidal configurations. The most recent grant covers the period May 1, 2000 to November 30, 2003, at a total cost of $\$ 227,090$. The research during this period covered the following topics: (1) the existence and properties of the MHD continua in plasma equilibria without spatial symmetry; (2) low frequency nonresonant current drive and nonlinear Alfvén wave effects; and (3) nonlinear electron acceleration by rf and random plasma waves. Results obtained during the funding period have contributed to the fundamental knowledge base of MHD activity in symmetric and asymmetric toroidal plasmas. Among the accomplishments of this research effort, the following are highlighted:

- Derivation of a third order ordinary differential equation that governs nonlinear current drive in the singular layers of the Alfvén continuum modes in axisymmetric toroidal geometry. Bounded solutions of this ODE implies a net average current parallel to the toroidal equilibrium magnetic field.

- Discovery of a new unstable continuum of the linearized MHD equation in axially periodic circular plasma cylinders with shear and incompressibility. This continuum, which we named "accumulation continuum" and which is related to ballooning modes, arises as discrete unstable eigenfrequency accumulate on the imaginary frequency axis in the limit of large mode numbers.

- Development of techniques to control nonlinear electron acceleration through the action of multiple coherent and random plasmas waves.

One graduate student who was supported by the grant during the past three years completed his Ph.D. research: Roberto Torasso. The title of his thesis is, "Nonlinear MHD Wave Processes in Toroidal Plasmas." His research focused on current drive resulting from MHD wave activity in axisymmetric toroidal geometry. Two important elements of this project were student participation and student training in plasma theory. 


\section{Overview of Research in Current Grant Period}

The past research on nonlinear MHD wave effects was a joint effort carried out by the PI and Roberto Torasso, who completed his Ph.D. research the University of Wisconsin with support provided by the current grant. In addition, the PI collaborated extensively with Dr. Albert Salat and Dr. Günther Spies of the Max-Planck-Institut für Plasmaphysik in Garching, Germany and with Dr. Václav Petržilka of the Czech Academy of Sciences. In this section, brief summaries of some of the important results from these studies is presented. More detailed information can be found in the publications listed Section 2-D.

\section{2-A. Studies of MHD Continuous Spectrum}

The shear Alfvén continuum of ideal MHD continues to receive considerable interest and attention because of its importance for plasma heating and current drive, and because of its effects on plasma stability. Current knowledge and understanding of the Alfvén continuum and its properties have arisen principally from studies of MHD wave propagation in equilibria with spatial symmetries, such as the one-dimensional cylindrical screw pinch and the two-dimensional toroidal tokamak. The shear Alfvén continuum in asymmetric equilibria has received minimal attention. In the absence of spatial symmetry, existence of MHD equilibria has not been clearly established. This lack of an existence proof and the mathematical difficulties associated with asymmetric geometries have likely impeded analytical studies of Alfvén wave behavior in three-dimensional plasmas.

During the current funding period, studies of the MHD continuum in three dimensional asymmetric geometry were carried out. The research focused on two aspects of the continuum problem: (1) the spatial singularities that characterize the Alfvén continuum modes, and (2) existence and composition of the continua. Papers $1-5$ listed in Sect. 2-D report results that were obtained in these studies. Papers 1, 2 and 3 deal with the spatial singularities of the Alfvén continuum modes in toroidal geometry. Paper 1, which was essentially completed in 1999, but has some overlap with the research in the current funding period, addresses the issue of the analytic form of the spatial singularities in axisymmetric toroidal geometry. Papers 2 and 3, on the other hand, are focused on the spatial singularities of general toroidal equilibria with a pressureless plasma. Papers 4 and 5 address the existence of continua in one dimensional equilibria in the limit of large mode numbers. Unstable continua, which we named "accumulation continua", are found. Paper 4 explore accumulation continua in circular cylinder plasma configuration, while paper 5 examines accumulation continua in a planar plasma slab under the influence of gravity. A key issue in paper 5 is the time evolution of unstable modes resulting from accumulation continua. Highlights of papers $1-5$ are discussed in Sects. 2-A-1 and 2-A-2. 
Paper 6 is more focused on the existence of orthogonal, magnetic field line coordinate systems, which we had to use in our spectrum studies. However, as we demonstrated in paper 6, such coordinate systems general do not exist. They exist only under special conditions, which are unlikely to be satisfied in toroidal geometry.

\section{2-A-1. Spatial singularities of continuum modes - - from papers 1 - 3}

Here, we review the theory that underlies the spatial singularities of the continuum eignemodes. Early studies of the MHD continuum modes in equilibria with two degrees of spatial symmetry (e.g. the 1-D sheet and screw pinches) have demonstrated that a logarithmic singularity characterizes the component of the plasma velocity along the normals to magnetic surface, $v_{\psi} \equiv \mathbf{v} \cdot \nabla \psi /|\nabla \psi| \sim \ln \left(\psi-\psi_{0}\right)$, where $\psi_{0}$ labels the resonant surface. In later years, a logarithmic singularity was also predicted for 2-D equilibria, such as an axisymmetric toroidal plasma.[2] It was also speculated that the logarithm would characterize the continuum modes in asymmetric toroidal geometries. [3, 4] During the current funding period, the spatial singularity of the continuum modes in 3-D equilibria without any continuous spatial symmetries was investigated. The results of this investigation are surprising because it was discovered that the logarithmic singularity characterizes the MHD continuum only in equilibria with certain types of spatial symmetries. It was found that the continuum modes are characterized by an essential singularity of the type $v_{\psi} \sim\left(\psi-\psi_{o}\right)^{i \tau}$, where $\tau$ is a real number, rather than the logarithm, even in axisymmetric toroidal geometry. Two particular configurations with the logarithmic singularity are an axisymmetric toroidal plasma with a purely poloidal magnetic field, and an axisymmetric toroidal plasma with up/down symmetry with respect to the equatorial plane.

The analysis of the continuum mode singularities in toroidal geometry is based on a matrix representation of the linearized MHD equations. Generalized magnetic surface coordinates are assumed, $\left(\psi, \ell_{1}, \ell_{2}\right)$, where $\ell_{1}$ and $\ell_{2}$ label points on magnetic surfaces, i.e. on surfaces of constant $\psi$. In terms of these coordinates, the linearized MHD equations can be reduced to a system two coupled matrix equations with the following structure,

$$
\begin{aligned}
& \mathcal{A} \mathbf{u}=\mathcal{B} \mathbf{w} \\
& \frac{\partial}{\partial \psi} \mathbf{w}=\mathcal{C} \mathbf{u}+\mathcal{D} \mathbf{w}
\end{aligned}
$$

where $\mathbf{u}$ and $\mathbf{w}$ are column vectors with, respectively, four and two elements. The elements of $\mathbf{w}$ are $v_{\psi} \equiv \mathbf{v} \cdot \nabla \psi$, the component of the plasma velocity normal to the magnetic surfaces, and the linearized total pressure $p+\mathbf{b} \cdot \mathbf{B}$. The elements of $\mathbf{u}$ are the four components of the linearized velocity and magnetic field in the magnetic surfaces. The four 
coefficients $\mathcal{A}, \mathcal{B}, \mathcal{C}$ and $\mathcal{D}$ are matrix differential operators that contain only derivatives with respect to the magnetic surface coordinates $\ell_{1}$ and $\ell_{2}$. The derivative with respect to $\psi$ does not appear in them. The operator $\mathcal{A}$ in particular contains only $\mathbf{B} \cdot \nabla$, which is the derivative along a force line of $\mathbf{B}$. Formally, Eqs. (1) and (2) are solved in two steps. First, Eq. (1), which contains no derivatives with respect to $\psi$, is solved for $\mathbf{u}$ on each magnetic surface, $\mathbf{u}=\mathcal{A}^{-1} \mathcal{B} \mathbf{w}$, where $\mathcal{A}^{-1}$ represents the inverse of $\mathcal{A}$. The solution is then substituted in Eq. (2), which results in an equation for $\mathrm{w}, \partial \mathrm{w} / \partial \psi=\mathcal{C} \mathcal{A}^{-1} \mathcal{B} \mathbf{w}+\mathcal{D} w$. The key question is, does $\mathcal{A}^{-1}$ exist on all magnetic surfaces? If $\mathcal{A}^{-1}$ does not exist on a particular magnetic surface, $\psi=\psi_{0}$, the equation governing $\mathbf{w}$ is singular, and its solutions will generally have spatial singularities about $\psi_{0}$. Existence of $\mathcal{A}^{-1}$ can be established by examining the null space of $\mathcal{A}$ defined by the homogeneous equation $\mathcal{A} u_{0}=0$ on a magnetic surface labeled by $\psi$. If the null space of $\mathcal{A}$ is not empty, i.e. if $\mathcal{A} \mathbf{u}_{0}=0$ has non-trivial solutions that satisfy periodic boundary conditions on a particular magnetic surface, $\mathcal{A}$ has no inverse on that surface. The question is then, what is the nature of the singular solutions of Eqs. (1) and (2) about the surfaces where the null space of $\mathcal{A}$ is not empty?

Our derivation of the spatial singularities is based on expansions of $\mathbf{u}$ and $\mathbf{w}$ about a surface $\psi_{0}$ where $\mathcal{A} \mathbf{u}_{0}=0$ is satisfied. A key assumption is that about $\psi_{0}$, the radial derivative $\partial / \partial \psi$ is large. This assumption can be incorporated in the analysis by introducing a small parameter $\epsilon$ that orders $\partial / \partial \psi$ as $\epsilon^{-1}$,

$$
\frac{\partial \mathbf{w}}{\partial \psi}=\epsilon^{-1} \frac{\partial \mathbf{w}}{\partial y} \quad y \equiv \frac{\psi-\psi_{0}}{\epsilon} \equiv \text { order } 1 \text { quantity }
$$

The vectors $\mathbf{u}$ and $\mathbf{w}$, and the equilibrium variables represented by $A$, are then expanded in $\epsilon$ (similar to Pao [2], Tataronis, Talmadge \& Shohet [3], Hameiri [4, 5]),

$$
\begin{aligned}
& \mathbf{u}=\mu_{0}(y) \mathbf{u}_{0}+\epsilon \mu_{1}(y) \mathbf{u}_{1}+\epsilon^{2} \mu_{2}(y) \mathbf{u}_{2}+\cdots \\
& \mathbf{w}=\epsilon\left[\nu_{0}(y) \mathbf{w}_{0}+\epsilon \nu_{1}(y) \mathbf{w}_{1}+\epsilon^{2} \nu_{2}(y) \mathbf{w}_{2}+\cdots\right] \\
& A=A_{0}+\epsilon y A_{1}+\epsilon^{2} y^{2} A_{2}+\cdots
\end{aligned}
$$

where the coefficients $\mathbf{u}_{k}, \mathbf{w}_{k}$ and $A_{k}$ do not depend on $y$. The spatial singularities that characterize $\mathbf{u}$ and $\mathbf{w}$ are determined by the leading factors $\mu_{0}(y)$ and $\nu_{0}(y)$ in the expansions. Substitution of these expansions in Eqs. (1) and (2) and grouping terms according to powers of $\epsilon$ lead to equations that determine the expansion coefficients,

$$
\begin{aligned}
& \text { order } \epsilon^{0}: \quad \mathcal{A}_{0} \mathbf{u}_{0}=0 \quad \mathbf{w}_{0}=\mathcal{C}_{0} \mathbf{u}_{0} \quad \nu_{0}^{\prime}=\mu_{0} \\
& \text { order } \epsilon^{1}: \quad \mu_{1} \mathcal{A}_{0} \mathbf{u}_{1}+y \mu_{0} \mathcal{A}_{1} \mathbf{u}_{0}=\nu_{0} \mathcal{B}_{0} \mathcal{C}_{0} \mathbf{u}_{0}
\end{aligned}
$$


Because $\mathcal{A}_{0} \mathbf{u}_{0}=0$ is satisfied on the surface labeled by $\psi_{0}$, Eq. (8), which determines $\mathbf{u}_{1}$, requires that a solvability condition be satisfied,

$$
y \mu_{0}=\sigma \nu_{0} \quad \sigma=\frac{\left\langle\mathbf{u}_{0}^{\dagger} \mathcal{B}_{0} \mathcal{C}_{0} \mathbf{u}_{0}\right\rangle}{\left\langle\mathbf{u}_{0}^{\dagger} \mathcal{A}_{1} \mathbf{u}_{0}\right\rangle}
$$

where $\mathbf{u}_{0}^{\dagger}$ is some null vector of the self-adjoint operator $\mathcal{A}_{0}$, and the brackets $\langle\cdots\rangle$ designate integration of the enclosed quantity over the singular magnetic surface $\psi_{0}$. Equations (7) and (9) form a system of coupled equations for $\mu_{0}$ and $\nu_{0}$,

$$
\nu_{0}^{\prime}=\mu_{0} \quad y \mu_{0}=\sigma \nu_{0}
$$

Two cases must be considered separately: $\sigma \neq 0$ and $\sigma=0$. If $\sigma \neq 0$, the solutions are

$$
\sigma \neq 0: \quad \mu_{0}=c_{0} y^{\sigma-1} \quad \nu_{0}=\frac{c_{0}}{\sigma} y^{\sigma}
$$

where $c_{0}$ is a constant. The singularity is therefore not logarithmic. If $\sigma=0$, the coupled equations in Eq. (10) do not apply, and the expansions of $\mathbf{u}$ and $\mathbf{w}$ must be altered. The result is a logarithmic singularity for $\mathbf{w}$ and a $\left(\psi-\psi_{0}\right)^{-1}$ singularity for $\mathbf{u}$ about $\psi_{0}$,

$$
\sigma=0: \quad \mathbf{u} \sim \ln \left(\psi-\psi_{0}\right) \mathbf{u}_{0}, \quad \mathbf{u} \sim\left(\psi-\psi_{0}\right)^{-1} \mathbf{u}_{0}
$$

The coefficient $\sigma$ thus determines the nature of continuum singularities. Expressions for $\sigma$ was derived for two cases: an axisymmetric toroidal plasma (in paper 1), and an asymmetric toroidal configuration with a pressureless plasma (in papers 2 and 3). Contained in these papers are evaluations of special configurations with specific spatial symmetries that have logarithmic singularities (meaning $\sigma=0$ ). In most configurations, however, we find that $\sigma$ given by Eq. (9) is imaginary, implying that singularities are of the essential type, $y^{i|\sigma|}$.

\section{2-A-2. The accumulation continua and ballooning modes in ideal magneto- hydrodynamics - - papers 4 and 5}

This research area of our program deals with the MHD spectra in the limit of large mode numbers. Ballooning modes fall into this class of wave modes. The results of our research are reported in paper 4 listed on page 19 of this document.

Past research on the continuous spectrum of the linearized MHD equations focused on the Alfvén continua and the Cusp continua. These stable continua pertain to perturbations with singularities across the equilibrium pressure surfaces. However, in addition to stable continua, unstable continua may exist. The unstable continua consist of accumulation points of eigenfrequencies of modes with large variation in the pressure surfaces, i.e. large mode numbers. Modes with large mode numbers are localized at magnetic field 
lines where the perturbations have finite variation. This class of modes are typically called "ballooning modes" because the eigenfunctions are believed to concentrate at those sections of the magnetic field line where its curvature is unfavorable for stability. This name, however, is not very concise because the modes may be present even if the curvature does not vary at all. We therefore have proposed the name "accumulation continua" for the associated continua.

It is a curious fact that modes with large mode numbers were first discovered in twodimensional equilibria, and that their theory was then generalized to three-dimensional configurations, but that little attention has been payed to one-dimensional ones, for example plane slab or circular cylinder that can be turned into a topological torus by imposing appropriate periodicity conditions. The various continua are actually most easily visualized in one-dimensional equilibria. This is because Fourier decomposition in the two ignorable coordinates leads to a system of ordinary differential equations in the remaining radial coordinate. The Alfvén continua and the Cusp continua correspond to singular points of this system. Since these continua are stable, the unstable parts of the subspectra pertaining to single Fourier modes, if there are any, are purely discrete. Unstable accumulation continua nevertheless arise if the union of these sub-spectra contains eigenfrequencies that approach accumulation points as some mode numbers become large.

Previous investigations of modes with large mode numbers focused on stability criteria and on the maximum growth rate in general geometries, rather than on the overall properties of the associated spectrum. The relevant asymptotic equations were written down in great generality, and in a rather abstract form, and it was noted that accumulation points arise that belong to the continuous spectrum. The nature of these accumulation continua, however, has to our knowledge not been studied. This is the object of the present research effort. Rather than focusing on generality, it is a goal of the research effort to gain a deeper understanding of unstable accumulation continua. This is a prerequisite for investigations of the effects and consequences of the accumulation continua.

We have therefore restricted attention to the simplest possible case, and leave generalizations to future investigations. The simplest equilibrium is a plane slab, but the modes of interest are trivial in this case because the magnetic field lines are straight. We thus consider a circular cylinder. We eliminate the magnetosonic waves and the associated Cusp continua by assuming incompressibility. We assume that the magnetic field has shear. We use cylindrical coordinates, and perform a singular layer analysis near the radius where the modes are localized. This yields a standard linear eigenvalue problem for a second order ordinary differential equation in a stretched radial coordinate ("singular layer equation"). This is in contrast to previous investigations in which the so-called "ballooning representation" was used to derive a fourth order equation along a magnetic 
field line. This equation, when specialized to incompressibility and to the geometry of a circular cylinder, is the Fourier transform of the singular layer equation. Both equations therefore describe the radial variation of the eigenfunctions rather than that along a field line. The variation along a field line is determined in higher orders of the expansion.

The singular layer equation yields novel results: The accumulation continua, if there are any, have no stable parts, their presence depends on Suydam's criterion (they are traced out by the eigenvalues of the singular layer equation as the singular radius runs through the interval where the criterion is violated), there is exactly one such continuum for any number of radial nodes, and this continuum is independent of the "parallel wave number" (which corresponds to the distance between nodes along a field line).

Basic equations: In a circular cylinder with magnetic rotation number,

$$
\mu(r)=\frac{R_{0} B_{\theta}}{r B_{z}}
$$

and magnetic field $\mathbf{B}(r)=\hat{\theta} B_{\theta}(r)+\hat{z} B_{z}(r)$, equilibrium quantities depend only on the radius $r\left(0 \leq r \leq r_{0}\right)$, while perturbing quantities also depend on the azimuthal angle $\theta$ and the axial coordinnate $z$ through a factor $\exp i\left(m \theta+n z / R_{0}\right)$, where the azimuthal mode number $m$ and the axial mode number $n$ are integers, and $(r, \theta, z)$ are cylindrical coordinates. One thus models a genuine torus with minor radius $r_{0}$ and major radius $R_{0}$. Replacing the perturbing plasma pressure $p$ by the perturbing total pressure $p_{*}=p+\mathbf{B} \cdot \mathbf{b}$, and then eliminating algebraically all dependent variables except $u=r u_{\tau}$ and $v=i \omega p_{*}$, one reduces the linearized equations to a system of two first order ordinary differential equations. This system is

$$
A u^{\prime}=-C_{1} u+r K^{2} v, \quad A v^{\prime}=C_{2} u+C_{1} v
$$

in the present incompressible limit. Its coefficients are

$$
\begin{aligned}
& A=\omega^{2} \rho-F^{2}, \quad K^{2}=\frac{m^{2}}{r^{2}}+\frac{n^{2}}{R_{0}^{2}} \\
& C_{1}=\frac{2 m B_{\theta} F}{r^{2}}, \quad C_{2}=\frac{1}{r} A\left[A+2 B_{\theta}\left(\frac{B_{\theta}}{r}\right)^{\prime}\right]-\frac{4 B_{\theta}^{2} F^{2}}{r^{3}},
\end{aligned}
$$

where

$$
F=\frac{m}{r} B_{\theta}+\frac{n}{R_{0}} B_{z}
$$

The coefficients are functions of $r$, and also depend on the frequency and the two mode numbers. Elimination of $v$ further reduces the system to the single second order equation

$$
\left(\frac{A}{r K^{2}} u^{\prime}\right)^{\prime}+C u=0, \quad C=\left(\frac{C_{1}}{r K^{2}}\right)^{\prime}-\frac{C_{1}^{2}+r K^{2} C_{2}}{r K^{2} A} .
$$


With a regularity condition at $r=0$ and a boundary condition at $r=r_{0}$, Eq. (18) constitutes an eigenvalue problem linear in the eigenfunction $u(r)$, but non-linear in the eigenvalue parameter $\omega^{2}$. Once Eq. (18) is solved, the remaining dependent variables can be computed algebraically. Both the solution $u$ and its derivative $u^{\prime}$ must be square integrable in order that the vector $\mathbf{u}$ be square integrable.

The asymptotic eigenvalue problem for modes with large $m$ and/or $n$ ("ballooning modes") has to our knowledge not been derived directly from eith Eqs. (14) or (18). Instead, the more general case of a resistive compressible plasma in an arbitrary threedimensional equilibrium was treated by Correa [D. Correa-Restrepo, Z. Naturforsch. A 37,848 (1982)]. An eigenvalue problem for a fourth order system of ordinary differential equations in a coordinate along this field line ("ballooning equations") was derived for perturbations localized at a closed magnetic field line (the same system was later independently obtained by other authors for the more special case of perfect conductivity). When specialized to the present case of perfect conductivity, incompressibility, and a circular cylinder, Correa's equations reduce to

$$
\left[\frac{d}{d \hat{y}}\left(1+\hat{y}^{2}\right) \frac{d}{d \hat{y}}-\hat{\alpha}_{2}-\hat{\gamma}^{2}\left(1+\hat{y}^{2}\right)\right] u_{1}-u_{2}=0, \quad\left(\frac{1}{\hat{\gamma}^{2}} \frac{d^{2}}{d \hat{y}^{2}}-1\right) u_{2}+\hat{\alpha}_{1}^{2} u_{1}=0
$$

The coefficients are

$$
\hat{\alpha}_{1}=\frac{2 \bar{\mu}^{2}}{R_{0} \bar{\mu}^{\prime}}, \quad \hat{\alpha}_{2}=\frac{2 \bar{P}^{\prime} \bar{\mu}^{2}}{\bar{r} \bar{B}_{z}^{2} \bar{\mu}^{\prime 2}}, \quad \hat{\gamma}^{2}=\frac{\gamma^{2} \rho \bar{B}^{2} \bar{\mu}^{2}}{\bar{B}_{\theta}^{2} \bar{B}_{z}^{2} \bar{\mu}^{\prime 2}},
$$

where a bar indicates that a quantity is taken at the field line under consideration, $\gamma=i \omega$ is the growth rate, Correa's independent variable $y$ has been replaced by $\hat{y}$ through

$$
2 \pi \bar{r} \bar{B}_{z} \bar{\mu}^{\prime} y=\bar{B} \bar{\mu} \hat{y}
$$

and Correa's dependent variables $F$ (not to be confused with the function $F$ of the present paper) and $D$ have been replaced by

$$
u_{1}=F, \quad u_{2}=\hat{\alpha}_{2} D
$$

The functions $u_{1}$ and $u_{2}$ must approach zero for large arguments; they are then square integrable.

The system is simpler than the corresponding system for arbitrary geometries in that it has constant coefficients. However, one wonders why it is of fourth order, because the original eigenvalue problem (18) for arbitrary mode numbers is of second order. This paradox is resolved below.

Singular layer equation: We have derived the asymptotic form of the second order equation (18) for large mode numbers, $m^{2}+n^{2} \gg 1$, and frequencies comparable with the 
Alfvén frequency, $\omega^{2} \rho r^{2} / B^{2}=O(1)$. The eigenfunctions are then localized at a radius where the function $F(r)$ remains finite in the limit $m^{2}+n^{2} \rightarrow \infty$ (it diverges everywhere else). We call this the "singular radius", and denote it by $\bar{r}$ (the singular radius should not be confused with a "resonant radius" $F=0$; a resonant radius would be defined only if $m$ and $n$ were fixed).

To be precise, we pick a radius $\bar{r}$ in the interval $0<\bar{r}<r_{0}$, construct a sequence $\left(m_{\nu}, n_{\nu}\right)$ of pairs of integers such that

$$
m_{\nu}^{2}+n_{\nu}^{2} \rightarrow \infty, \quad n_{\nu}+m_{\nu} \bar{\mu} \rightarrow \kappa,
$$

as $\nu \rightarrow \infty$, with $\bar{\mu}=\mu(\bar{r})$ and some given number $\kappa$, and put $(m, n)=\left(m_{\nu}, n_{\nu}\right)$. The radius $\bar{r}$ is arbitrary, except that the shear must not vanish there, $\mu^{\prime}(\bar{r}) \neq 0$. In particular, $\bar{\mu}$ need not be rational (localization is not restricted to closed field lines). While modes with large made numbers correspond to large $\nu$, the accumulation continua correspond to the limit $\nu \rightarrow \infty$. We shall take this limit. It is demonstrated in paper 4 that the number $\kappa$ is related to the longitudinal wave number $k_{\|}=2 \pi / l_{\|}$(where $l_{\|}$is the periodicity length along a field line at the singular radius) by $\kappa^{2}=\left(R_{0}^{2}+\bar{\mu}^{2} \bar{r}^{2}\right) k_{\|}^{2}$.

Since $F \sim B K$ everywhere except near the singular radius, one must distinguish outer regions where $F^{2} \gg B^{2} / R_{0}^{2}$ and a singular layer where $F^{2} \ll K^{2} B^{2}$. The overlap regions $B^{2} / R_{0}^{2} \ll F^{2} \ll K^{2} B^{2}$ are not empty because $K R_{0} \gg 1$. In the outer regions, an eikonal expansion of the solutions of Eq. (18) yields

$$
u \cong|K r / F|^{1 / 2} \exp \left( \pm \int d r K\right)
$$

while in the overlap regions, the solutions (24) reduce to

$$
u(r) \cong|r-\bar{r}|^{-1 / 2} \exp [ \pm K(\bar{r})(r-\bar{r})] .
$$

Since some linear combinations of these must match the solution in the singular layer in both outer regions, the regularity condition, together with any boundary condition of interest, require that the solution of the singular layer equation approaches zero at both sides. Equation (25) shows that it then decays exponentially within a distance $O\left(K^{-1}\right)$, and is square integrable.

The following simplifications are made in the singular layer:

1. Since equilibrium quantities vary only little within the layer, they are taken at $r=\bar{r}$ (as before, we indicate this with a bar). This applies also to the derivative $F^{\prime}(r)$. It does not apply to the function $F(r)$ itself, because this varies strongly.

2. Since $F(\bar{r}) \ll \bar{r} \bar{F}^{\prime}$, only the quantity $F$ must be differentiated in the first term of the coefficient $C$ in Eq. (18). 
3. The relation $n / m \cong-\bar{\mu}$ is used to express the mode numbers $m$ and $n$ in terms of equilibrium quantities.

4. The radius $r$ is replaced by the stretched variable $x=F(r)$.

Equation (18) then acquires the form

$$
\alpha_{0}^{2} \frac{d}{d x}\left(\omega^{2} \rho-x^{2}\right) \frac{d}{d x} u+\left(\alpha_{2}+\frac{\alpha_{1}^{2} \omega^{2} \rho}{\omega^{2} \rho-x^{2}}-\omega^{2} \rho+x^{2}\right) u=0 .
$$

The constant coefficients $\alpha_{i}$ can be cast into the form

$$
\alpha_{0}=\frac{\bar{B}_{\theta} \bar{B}_{z} \bar{\mu}^{\prime}}{\bar{B} \bar{\mu}}, \quad \alpha_{1}=\frac{2 \bar{B}_{\theta}^{2}}{\bar{r} \bar{B}}, \quad \alpha_{2}=\frac{2 \bar{B}_{\theta}^{2} \bar{P}^{\prime}}{\bar{r} \bar{B}^{2}} .
$$

The boundary conditions are $u \rightarrow 0$ as $\hat{x} \rightarrow \pm \infty$. Equation (26) has no positive eigenvalues $\omega^{2}$. In other words, no eigenvalues are superimposed upon the Alfvén continua which now cover the entire real axis. We may therefore assume $\omega^{2}<0$, and replace the imaginary frequency $\omega$ by the real growth rate $\gamma=|\omega|$.

We non-dimensionalize the problem by introducing the non-dimensional independent variable $\hat{x}$ and the non-dimensional growth rate $\hat{\gamma}$ by

$$
\gamma^{2} \rho \hat{x}^{2}=x^{2}, \quad \hat{\alpha}_{0}^{2} \hat{\gamma}^{2}=\rho \gamma^{2}
$$

Equation (26) is then

$$
\frac{d}{d \hat{x}}\left(1+\hat{x}^{2}\right) \frac{d}{d \hat{x}} u-\left(\hat{\alpha}_{2}+\frac{\hat{\alpha}_{1}^{2}}{1+\hat{x}^{2}}\right) u=\hat{\gamma}^{2}\left(1+\hat{x}^{2}\right) u
$$

with non-dimensional coefficients

$$
\hat{\alpha}_{1}=\alpha_{1} / \alpha_{0}, \quad \hat{\alpha}_{2}=\alpha_{2} / \alpha_{0}^{2}
$$

The coefficients (30) are explicitly given in Eqs. (20). Equation (29) is simpler than the original equation (18) in that it is linear in the eigenvalue parameter, in that it is independent of any mode numbers, and in that it depends on only two equilibrium parameters.

To show the equivalence to the "ballooning equations" (19), we write Eq. (29) as a similar system,

$$
\left[\frac{d}{d \hat{x}}\left(1+\hat{x}^{2}\right) \frac{d}{d \hat{x}}-\hat{\alpha}_{2}-\hat{\gamma}^{2}\left(1+\hat{x}^{2}\right)\right] v_{1}-v_{2}=0, \quad\left(1+\hat{x}^{2}\right) v_{2}-\hat{\alpha}_{1}^{2} v_{1}=0
$$

We introduce the Fourier transforms $u_{i}(\hat{y})$ of the functions $v_{i}(\hat{x})$ by

$$
v_{i}(\hat{x})=\int d \hat{y} u_{i}(\hat{y}) \exp (-i \hat{\gamma} \hat{x} \hat{y})
$$


where the integration is along the entire real axis. Since differentiation and multiplication then transform into each other,

$$
\frac{d}{d \hat{x}} \rightarrow-i \hat{\gamma} \hat{y}, \quad \hat{x} \rightarrow \frac{1}{i \hat{\gamma}} \frac{d}{d \hat{y}}
$$

and since the two operators $\hat{y}(d / d \hat{y})$ and $(d / d \hat{y}) \hat{y}$ commute, it is readily verified that the systems (31) and (19) transform into each other. The two systems therefore have the same eigenvalues, and the eigenfunctions are Fourier transforms of each other. Since the singular layer equation describes the radial variation of the perturbation rather than that along the field lines, the same is true for the "ballooning equations".

Eigenvalues: The following spectral properties of the non-dimensional singular layer equation (29) are derived in paper 4:

1. The equation has no negative eigenvalues $\hat{\gamma}^{2}$.

2. If there are any eigenvalues at all, $\hat{\gamma}^{2}>0$ and then they have the Sturmian property (note that $\hat{\gamma}^{2}>0$ corresponds to $\omega^{2}>0$ ).

3. There are either infinitely many positive eigenvalues that accumulate at the origin $\hat{\gamma}^{2}=0$, or none at all, depending on whether Suydam's criterion

$$
P^{\prime}+\frac{r B_{z}^{2} \mu^{\prime 2}}{8 \mu^{2}} \geq 0
$$

is violated or satisfied at the singular radius. This condition is equivalent to $S \geq 0$, with $S=\hat{\alpha}_{2}+\frac{1}{4}$.

Planar sheet pinch: Accumulation continua was found here in a circular cylinder plasma. The governing normalized equation of the continua is Eq. (29). A simpler one dimensional plasma model that also has accumulation continua is the planar sheet pinch under the influence of gravity. Gravity produces effects analagous to the curvature effects of the helically shaped magnetic field of the circular cylinder. For the sheet pinch configuration, Cartesian coordinate $(x, y, z)$ are assumed, where $x$ is the effective radial coordinate, and $y$ and $z$ are magnetic surface coordinates. Because the equilibrium is independent of $y$ and $z$, Fourier modes can be assumed, whereby perturbations depend on $y$ and $z$ through the factor $\exp \left(k_{y} y+k_{z} z\right)$, with $k_{y}=m_{y} / R_{y}$ and $k_{z}=m_{z} / R_{z}$. The constants $R_{y}$ and $R_{z}$ are minor and major radii of a geometrically equivalent torus, and $m_{y}$ and $m_{z}$ are integers. Let $u(x)$ be the $x$-component of the fluctuating plasma velocity. Then, $u(x)$ is governed by the following ODE,

$$
\frac{d}{d x}\left[\left(\rho \omega^{2}-F^{2}\right) \frac{d u}{d x}\right]-k^{2}\left(\rho \omega^{2}-F^{2}+\rho^{\prime} \Phi^{\prime}\right) u=0
$$


Here,

- $k^{2}=k_{y}^{2}+k_{z}^{2}$

- $F=\mathbf{k} \cdot \mathbf{B}=\frac{m_{y}}{R_{y}} B_{y}+\frac{m_{z}}{R_{y}} B_{z}$

- $\Phi(x)$ is the gravitational potenial,

- $\Phi^{\prime}(x)$ denotes derivative with respect to $x$.

In the limit of large modes numbers $m_{y}$ and/or $m_{z}$, Eq. (35) reduces to the following normalized equation in the singular layer,

$$
\frac{d}{d \phi}\left[\left(\rho \gamma^{2}+\phi^{2}\right) \frac{d u}{d \phi}\right]-\frac{L^{2}}{B^{2}}\left(\rho \gamma^{2}+\phi^{2}-\rho^{\prime} \Phi^{\prime}\right) u=0
$$

where $\phi=F(x), L$ is the shear length, and $\gamma^{2}=-\omega^{2}$. Equation (36) is mathematically equivalent to Eq. (29) if $\alpha_{1}^{2}$ is set equal to zero. Therefore, the continuum properties that we found with Eq. (29) applies also to Eq. (36) for the sheet pinch in the presence of gravity. Currently, we are using this model and Eq. (36) to explore the time evolution of perturbations that results from accumulation. This is a topic that will receive further attention in the new funding period.

Summary: We have derived a second order ODE that determines the accumulation eigenfrequencies in the limit of infinitely large mode number. This equation has no eigenvalues at all if Suydam's criterion is met at the singular radius, but infinitely many eigenvalues exist if the criterion is violated. When labeled by the radial mode number $l(0 \leq l<\infty)$, the eigenvalues are subject to $0<\hat{\gamma}_{l+1}^{2}<\hat{\gamma}_{l}^{2}$ (Sturmian property), and $\hat{\gamma}_{l}^{2} \rightarrow 0$ as $l \rightarrow \infty$ (Suydam accumulation at the origin). Each eigenvalue is a function of the singular radius, because it depends on equilibrium quantities at the singular radius through the two parameters $\hat{\alpha}_{1}$ and $\hat{\alpha}_{2}$. This function traces out a continuum as the radius runs through the interval in which Suydam's criterion is violated. There is one such accumulation continuum for every radial mode number.

The continuous spectrum thus consists of the Alfvén continua and the accumulation continua, and there are no other continua in the present incompressible case. The two classes of continua have the common origin that the magnetic field lines are real characteristics. The singular gradient of the perturbation is always perpendicular to a field line, and different continua merely correspond to different orientations of this gradient. The reason why the two classes are nevertheless profoundly different is that the Alfvén continua correspond to global magnetic surfaces (i. e. the pressure surfaces), while the accumulation continua correspond to magnetic surfaces that cannot be continued in the 
large in a toroidal equilibrium with shear. The unity of the two classes therefore manifests itself only if all magnetic surfaces are global, e. g. in equilibria with open ends (no torus) or with closed field lines (no shear).

Violation of Suydam's criterion is necessary and sufficient for the presence not only of the accumulation continua, but also of an infinite set of unstable eigenvalues. This set consists of "Suydam modes" and "ballooning modes". The former have a large number of nodes across the pressure surfaces, and the latter have a large number of nodes in the pressure surfaces. If both numbers are large, then the mode has both names. Suydam instability and the presence of accumulation continua, however, are no more equivalent in equilibria with less symmetry. This is because Mercier's criterion (which generalizes Suydam's criterion) is in general less restrictive than the "ballooning criterion". Unstable accumulation continua (and hence also unstable "ballooning modes") thus may occur even if Mercier's criterion is met. A study of the continuous spectrum in three dimensional equilibria with zero plasma pressure shows that the accumulation continua have stable parts in general.

\section{2-B. Low Frequency Nonresonant Current Drive and Nonlinear Alfvén Wave Efects - - Torasso's Ph.D. Thesis, papers 8 and 9}

In 1992, with DOE support, the PI and two graduate students, Richard Mett and Shahid Rauf, undertook a study of rf current drive based on the low frequency Alfvén wave. The research effort was very successful. It led to two $\mathrm{Ph}$.D. theses that addressed fundamental issues of rf current drive. A limitation of the research, however, was that emphasis was not placed on geometry. The focus was more on the key physics issues. Geometry, however, can significantly impact the effectiveness of this form of current drive.

During the current funding period, an investigation of $\mathrm{rf}$ current drive in axisymmetric toroidal geometry was undertaken by Roberto Torasso. He used his work on this topic to satisfy the research requirement for his Ph.D. degree at the University of Wisconsin.

A feature of the current that emerges from past studies is that the induced current is localized to small regions about the Alfvén wave resonant surface, which are the magnetic surfaces. Because of the localization, the induced current can be used to control locally the current density profile and modify the equilibrium magnetic field for improvement of confinement in toroidal fusion devices. For instance, gradients in the current density aligned with the equilibrium magnetic field form an energy source for magnetic fluctuations, which can affect plasma confinement. Therefore, it is of interest and importance to find ways to flatten the current density profile. The theory of current drive localized 
to the Alfvén resonance region developed in this research program might play a role in controlling the current density profile.

The theory of current drive in the Alfven resonance region relies on the continuous spectrum of the linearized equations of ideal magnetohydrodynamics (MHD). The identification of the continuous spectrum is tied to singular solutions of the linearized ideal MHD equations. In axisymmetric toroidal geometry the equilibrium configuration is two dimensional and the magnetic field lines lie on a set of closed nested magnetic flux surfaces. The frequencies associated with the modes are discrete on any particular flux surface but they cover a continuous range of values as the flux surface is varied from the magnetic axis to the plasma edge, thereby forming the continuum. The presence of the continuum is connected to the existence of singular solutions of a system of coupled second order partial differential equations on each magnetic flux surface.

The nature of the singularities of the modes is well established in cylindrical geometry. For a compressible plasma, the radial singularities are logarithmic, whereas the singularities become essential and of undulatory type, for an incompressible plasma. However, it was discovered during an earlier phase of this research program that the commonly held view of a logarithmic singularity for both a compressible and incompressible plasma in axisymmetric toroidal geometry is only valid for certain equilibrium configurations, for instance if the plasma cross section is up/down symmetric. In general, an essential singularity with an undulatory variation characterizes the continuum modes.

The physical mechanism of the current production in the Alfvén resonance region is based on the nonlinear mixing of linear shear Alfvén waves. Plasma is a nonlinear medium. If linear shear Alfvén waves are excited in it, the action of the nonlinearities causes the shear Alfvén waves to mix. A consequence of this nonlinear wave mixing process is the generation of a steady state current.

Torasso developed a theory for steady state current in the Alfvén resonance region for an incompressible resistive plasma in axisymmetric toroidal geometry. The steady state current is enhanced in the Alfvén resonance region, where the amplitudes of the waves have steep gradients. Torasso finds that a net total current emerges at second order in an expansion that assumes a toroidal configuration with a large aspect ratio.

Summary of analysis: Details of the analysis made by Torasso can be found in his Ph.D. thesis, which is listed on page 19, and papers 8 and 9 listed on page 20 . He we present a few key highlights.

Alfvén wave current drive is described by the nonlinear resistive MHD equations with 
incompressibility as the equation of state,

$$
\begin{aligned}
& \rho\left[\frac{\partial \mathbf{V}}{\partial t}+(\mathbf{V} \cdot \nabla) \mathbf{V}\right]=\frac{1}{\mu_{0}}(\nabla \times \mathbf{B}) \times \mathbf{B}-\nabla P \\
& \frac{\partial \mathbf{B}}{\partial t}=\nabla \times(\mathbf{V} \times \mathbf{B})+\frac{\eta}{\mu_{0}} \nabla^{2} \mathbf{B} \\
& \nabla \cdot \mathbf{V}=0
\end{aligned}
$$

where $\mathbf{V}$ is the plasma velocity, $\mathbf{B}$ is the magnetic field, $\rho$ is the plasma mass density, $P$ is the plasma pressure, $\eta$ is the plasma resistivity, and $\mu_{0}$ is the vacuum permeability. The effects of compressibility will be evaluated at a later time in the program. The underlying physics of the current drive process can be described in terms of the time-averaged Ohm's law,

$$
\langle\mathbf{E}\rangle+\langle\mathbf{V} \times \mathbf{B}\rangle=\eta\langle\mathbf{J}\rangle
$$

where $\mathrm{V}$ and $\mathrm{B}$ are, respectively, the velocity field and the magnetic field of the Alfvén wave, and $\mathbf{J}$ is the induced plasma current. The magnetic field associated with $\langle\mathbf{J}\rangle$ can derived from Ampère's law, $\mu_{0} \mathbf{J}=\nabla \times \mathbf{B}$. In essence, the current produced by the Alfvén wave is an rf dynamo process, where the fluctuating velocity and magnetic fields come from the propagating rf wave.

The Alfvén wave induced current is computed with Ohm's law and Faraday's law,

$$
\begin{aligned}
& \mathbf{E}+\mathbf{V} \times \mathbf{B}=\eta \mathbf{J} \\
& \nabla \times \mathbf{E}=-\frac{\partial \mathbf{B}}{\partial t}
\end{aligned}
$$

where $\eta$ is the resistivity. Orthogonal toroidal coordinates $(\psi, \chi, \phi)$ and an orthonormal set of bases vectors $\left(\mathbf{e}_{\psi}, \mathbf{e}_{\|}, \mathbf{e}_{\perp}\right)$ are assumed.[6] Here,

- $\psi=$ const. defines next toroidal magnetic surfaces, $\phi$ is the toroidal angle, and $\chi$ is the poloidal angle that satisfies $\nabla \psi \cdot \nabla \chi=0$;

- $\mathbf{e}_{\psi}$ is a unit vector normal to magnetic surfaces $\psi=$ const; $\mathbf{e}_{\|}$is a unit vector parallel to the equilibrium magnetic field; and $\mathbf{e}_{\perp}$ is a unit vector perpendicular to $\mathbf{e}_{\psi}$ and $\mathbf{e}_{\|}$

Vector fields, $\mathbf{B}_{1}$, for example, have components $\left(B_{1 \psi}, B_{1 \|}, B_{1 \perp}\right)$ with respect to these bases vectors, i.e.,

$$
\mathbf{B}_{1}=B_{1 \psi} \mathbf{e} \psi+B_{1 \|} \mathbf{e}_{\|}+B_{1 \perp} \mathbf{e}_{\perp}
$$


The analytic process is now straightforward but somewhat tedious. Only results will be quoted here. Details can be found in Torasso's thesis. Vector components in Eqs. (41) and (42) are expanded according to a scheme that represents mode beating: fluctuations with phase $\Phi$ beat to produce higher and lower harmonics of $\Phi$. The expansion has the form,

$$
u=u_{0}+\delta u_{1} e^{i \Phi}+\delta^{2}\left[u_{2}^{(0)}+u_{2}^{(2)} e^{i 2 \Phi}\right]+\cdots+\text { c.c. }
$$

where $\Phi(\equiv n \phi-\omega t)$ is the phase of the order 1 fields, $n$ is the toroidal mode number, $\omega$ is the frequency, and the parameter $\delta$, which is assumed small, is a measure of the wave amplitudes of order 1 fields. In Eq. (44),

- $\delta u_{1} e^{i \Phi}$ represents the Alfvén wave with phase $\Phi$. The linearized MHD equations govern this quantity.

- $\delta^{2}\left[u_{2}^{(0)}+u_{2}^{(2)} e^{i 2 \psi}\right]$ represents second order effects with zero harmonic and second harmonic terms that the plasma nonlinearities produce.

It is the nonlinearly generated second order term $\delta^{2} u_{2}^{(0)}$ that is of interest to us because it is a time-averaged term produced by the Alfvén wave. One now substitutes the expansion of Eq. (44) in Eqs. (41) and (42) and groups terms according to $\delta$. One finds then in this process the following expression for the induced second order current density $\mathbf{J}_{2}^{(0)}$,

$$
\mathbf{J}_{2}^{(0)}=-\frac{i \omega}{\eta}\left(\mathbf{B}_{1} \times \boldsymbol{\xi}_{1}^{*}-\mathbf{B}_{1}^{*} \times \boldsymbol{\xi}_{1}\right)
$$

where $\boldsymbol{\xi}_{1}$ is the displacement vector corresponding to the velocity perturbation $\mathbf{V}_{1}, \mathbf{V}_{1}=$ $i \omega \boldsymbol{\xi}_{1}$. We are interested here specifically in the component of $\mathbf{J}_{2}^{(0)}$ parallel to the equilibrium magnetic field,

$$
J_{2 \|}^{(0)}=\mathbf{J}_{2}^{(0)} \cdot \mathbf{e}_{\|}=-\frac{i \omega}{\eta}\left(B_{1 \psi} \xi_{1 \perp}^{*}-B_{1 \perp}^{*} \xi_{1 \psi}-c . c .\right)
$$

At this stage, the resistivity is assumed small. One then introduces the following scalings for the components of $\mathbf{B}$ and $\boldsymbol{\xi}$ with respect to $\eta$ in the small resistive layer about the Alfvén resonance surface,

$$
\begin{aligned}
B_{1 \psi} & =B_{10 \psi}+\eta^{1 / 3} B_{11 \psi}+\cdots \\
B_{1 \|} & =\eta^{-1 / 3} B_{10 \|}+B_{11 \|}+\cdots \\
B_{1 \perp} & =\eta^{-1 / 3} B_{10 \perp}+B_{11 \perp}+\cdots \\
\xi_{1 \psi} & =\xi_{10 \psi}+\eta^{1 / 3} \xi_{11 \psi}+\cdots \\
\xi_{1 \|} & =\eta^{-1 / 3} \xi_{10 \|}+\xi_{11 \|}+\cdots \\
\xi_{1 \perp} & =\eta^{-1 / 3} \xi_{10 \perp}+\xi_{11 \perp}+\cdots
\end{aligned}
$$


Substitute these expansions in Eq. (46),

$$
J_{2 \|}^{(0)}=-\frac{i \omega}{\eta^{4 / 3}}\left(B_{10 \psi} \xi_{10 \perp}^{*}-B_{10 \perp}^{*} \xi_{10 \psi}-c . c .\right)
$$

Equation (53) shows that $J_{2 \|}^{(0)}$ scales as $\eta^{-4 / 3}$, which is considerably stronger than the scaling found in Cartesian geometry. Equation (53) is completed by expressing the components of $\mathbf{B}_{10}$ in terms of the components of $\boldsymbol{\xi}_{10}$, which can be done by invoking Faraday's law, Eq. (42). Analysis of the resulting expressions reveals that bounded solutions of the following ordinary differential determines the magnetic and velocity fields in the small resistive layer about the Alfvén resonance surface, $\psi=\psi_{0}$,

$$
\frac{\partial^{3} u}{\partial \sigma^{3}}+\alpha \sigma \frac{\partial u}{\partial \sigma}+\beta u=0
$$

where $\alpha$ and $\beta$ are complex constants expressible in terms of equilibrium quantities, and $\sigma$ is a stretched variable in the resistive layer,

$$
\sigma=\frac{\psi-\psi_{0}}{\eta^{1 / 3}}
$$

where $\eta$ is the resistivity. Solutions of Eq. (54) belong to the class of "generalized hypergeometric functions," which have been investigated by Langer [see R.E. Langer, Duke Math. J. 22, 525 (1955)]. A complication in the solution of Eq. (54) is the Stokes' phenomenon, which leads to different asymptotic forms of $u(\sigma)$ in different regions of the complex $\sigma$ plane. We are presently investigating the consequences of the Stokes' phenomenon in our applications in the limit of small resistivity, corresponding to $|\sigma| \rightarrow \infty$. Details of this analytic process and further results are contained in Torasso's thesis and in paper 9 listed on page 20 .

\section{2-C. Nonlinear Electron Acceleration by RF and Random Fields - - papers 11 and 12}

During the past funding period, the PI collaborated extensively with Dr. Václav Petržílka of the Czech Academy of Sciences on the subject of nonlinear RF electron acceleration in plasmas. This topic is important because electron acceleration can lead to charge density perturbations and stationary electric fields that can strongly alter plasma properties. Our past studies focused on two modes of RF plasma waves that produce acceleration: the lower hybrid $(\mathrm{LH})$ mode and the lower frequency helicon mode. Significant electron acceleration was found in each case. An issue that we addressed in our past studies is the role played by random electric fields in the acceleration process. We found that electron acceleration produced by coherent $\mathrm{rf}$ waves can be significantly enhanced when random electric fields are present.[8] Moreover, the width of the acceleration layer, where 
the acceleration process takes place, can be increased by the random fields. Because of the enhanced acceleration, electrons can be expelled from the antenna region, resulting in strong radial and toroidal stationary electric fields. These induced electric fields can produce plasma vortices front of the antenna structure [9]. Vortices, which results in poloidal plasma inhomogeneities in front of the grill, can significantly impact RF wave coupling and thermal loading of the walls. There is evidence of these induced vortices from the ASDEX [10] and TdeV [11] tokamak experiments. Random electric and magnetic fields, which are intrinsic to most plasma environments, can appear, for example, near antennas that excite RF waves. Two possible sources of random fields are the following: (1) nonlinear parametric decay of waves, and (2) scattering of waves from plasma density fluctuations.

Another wave configuration that we explored during the current funding period consists of multiple rf waves with and without random fields. In a recent analysis by V. Petržílka and L. Krlín of the Czech Academy of Sciences, in collaboration with the PI, we performed a 3-d two-fluid analysis of perturbations arising in plasmas because of generation of fast electrons in two crossing laser beams. It is assumed that the electrons are accelerated in a single plane laser beam, and an additional (secondary) perpendicularly propagating plane laser beam with a randomized phase is present [1]. The laser frequencies and laser beam electric field intensities chosen for the computations of the primary electron acceleration match the parameters available at the Prague Asterix Laser System (PALS). The acceleration mechanism is analogous to the electron acceleration due to dephasing of the electron motion in a plane transverse laser beam by random kicks, as found in. The random kicks are now provided by the additional randomized laser beam, which propagates perpendicularly to the direction of propagation of the main laser beam, and which is polarized along the direction of the main laser beam propagation. The power flux intensity of the additional beam can be typically much lower than the power flux intensity of the main laser beam. As the accelerated electrons are pushed away, they leave the heavier ions behind, and thus an electrostatic field and consequent plasma flows are generated. For the modeling, we developed an advanced version of our numerical code [2]. We originally developed this 3-d two-fluid numerical code for analysis of local generation of fast electrons by lower hybrid waves in a thin layer just in front of the grill mouth in large tokamaks. The code now allows propagation of the density perturbation across the boundary of the primary electron acceleration region. By using test particle simulations of electron acceleration for parameters relevant to conditions available by the PALS laser, it is possible to determine an effective potential $\mathrm{W}$, which expels and accelerates the ion fluid. We found that rather significant plasma density perturbations, electrostatic charge separation fields and plasma flows can develop as a consequence of the primary electron 
acceleration and of the ensuing Coulomb separation potential We also present the dependence of the power flux of the accelerated plasma fluid on the intensity of the laser beams. Results from this research project will be reported at the 30th European Physical Society Conference on Controlled Fusion and Plasma Physics (St. Petersburg, Russia, 7 - 11 July 2003).

Results of these studies have been reported at a variety of European and American conferences. All conference presentations are listed in Sect. 2-D. Reports that will be submitted for publication are now under preparation.

\section{2-D. Publications}

Results of the research that has been carried during the current funding period have been reported in several publications and conferences. This material is documented in this section.

\section{Ph.D. Thesis}

1. Roberto Torasso, "Non-Linear MHD Wave Proceses in Toroidal Plasmas," Ph.D. Dissertation, Department of Nuclear Engineering and Engineering Physics, University of Wisconsin - Madison (2001).

\section{Papers}

\section{Alfvén Resonance and MHD Continuum}

1. A. Salat and J.A. Tataronis, "Radial Dependence of Magnetohydrodynamic Continuum Modes in Axisymmetric Toroidal Geometry," Phys. Plasmas 6, 3207 (1999) - - also identifed in 1999 proposal.

2. A. Salat and J.A. Tataronis, "Shear Alfvén mode resonances in nonaxisymmetric toroidal low-pressure plasmas. I. Modes equations in arbitrary geometry," Phys. Plasmas 81200 (2001).

3. A. Salat and J.A. Tataronis, "Shear Alfvén mode resonances in nonaxisymmetric toroidal low-pressure plasmas. II. , "Singular modes in the shear Alfvén continuum," Phys. Plasmas 81207 (2001).

4. G.O. Spies and J.A. Tataronis, "The accumulation continua in ideal magnetohydrodynamics," Phys. Plasmas 10413 (2003). 
5. G.O. Spies and J.A. Tataronis, "Effect of unstable continuous spectrum in ideal magnetohydrodynamics," under preparation and to be submitted to Phys. Plasmas.

\section{MHD Equilibrium}

6. A. Salat and J.A. Tataronis, "On the existence of Orthogonal, Magnetic Field Line Oriented Coordinate Systems," J. Geophys. Research A 105, 13055 (2000).

\section{Nonlinear Alfvén Wave Current Drive}

7. S. Rauf and J.A. Tataronis, "Steady-State Magnetic Field in the Alfvén Resonance Region," Phys. Rev. E 52, 4311 (1995) - - also identifed in 1999 proposal.

8. R. Torasso, J.A. Tataronis and C.K. Phillips, "Radial dependence of magnetohydrodynamic modes in axisymmetric toroidal geometry in the incompressible limit," submitted to Phys. Plasmas.

9. R. Torasso and J.A. Tataronis, "Alfvén wave current drive in axisymmetric toroidal geometry," submitted to Phys. Plasmas.

\section{Ponderomotive force effects}

10. L. Krlín, R. Klíma, P. Pavlo, V. Petržílka, V. Fuchs, V. Svoboda and J.A. Tataronis, "Transport of Intensive LH Pulses into the Tokamak Plasma," J. Plasma Phys. 62, 203 (1999) - - also identified in 1999 proposal.

11. V. Petržílka, J.A. Tataronis and L. Krlín, "Random Field Effects on Electron Acceleration in Crossed Laser Beams," paper published in Czech. J. Phys. 50, Suppl. 3 , p. $202(2000)$.

12. V. Petržílka, L. Krlín and J.A. Tataronis, "Electron Acceleration in a Plane Laser Beam," paper published in Czech. J. Phys. 52 , Suppl. 3, p. 279 (2002).

\section{Conference Presentations}

2000

- International Sherwood Theory Conference, Los Angeles, CA 27 - 29 March 2000. 
1. J.C. Wright, J.C. Prager and J.A. Tataronis, "Nonlinear MHD Alfven Wave Computations in Cylindrical Geometry," abstract $1 \mathrm{C} 05$ in Conference Proceedings.

- 19th Symposium on Plasma Physics and Technology, Prague, Czech Republic, 12 - 16 June 2000.

1. V. Petržílka, J.A. Tataronis and L. Krlín, "Random Field Effects on Electron Acceleration in Crossed Laser Beams," paper published in Czech. J. Phys. 50, Suppl. 3, p.202-206 (2000).

- 26th European Conference on Laser Interaction with Matter (ECLM 2000), Prague, Czech Republic, 12 - 16 June 2000.

1. V. Petržílka, J.A. Tataronis and L. Krlín, "Enhancement of Electron Acceleration in Laser Wakefields," p. 296 of Conference Proceedings.

- Euroconference on Ultraintense Laser Interactions and Application (ULIA2), Pisa, Italy, 29 September - 3 October 2000.

1. V. Petržílkaand J.A. Tataronis, "Synergetic Effects of Transverse, Longitudinal and Random Electric Fields on Electron Acceleration in Laser Plasmas," p. 44 of Conference Proceedings.

- 42nd Annual Meeting of the Plasma Physics Division of the American Physical Society, Québec, Canada, 23 -27 October 2000.

1. J.C. Wright, S.C. Prager and J.A. Tataronis, "Simulations of Alfven Wave Current Drive in Cylindrical Geometry" - abstract published in Bull. Am. Phys. Soc. 45, 50 (2000).

2. R. Torasso, J.A. Tataronis and C.C. Hegna, "Non-Linear MHD Alfven Wave Current Drive in Axisymmetric Toroidal Plasmas" - abstract published in Bull. Am. Phys. Soc. 45, 51 (2000).

3. J.A. Tataronis, V. Petržílka and L: Krlín, "Two Dimensional Electron Acceleration in Laser Wakefields" - abstract published in Bull. Am. Phys. Soc. 45, $310(2000)$.

$-2001$

- International Sherwood Theory Conference, Santa Fe, NM 2 - 4 April 2001. 
1. J.A. Tataronis, R. Torasso, A. Salat and C. Phillips, "Ideal MHD Continuum Modes in General Toroidal Geometry," abstract 1C39 in Conference Proceedings.

2. R. Torasso, J.A. Tataronis and C.C. Hegna, "Non-Linear MHD Alfven Wave Current Drive in Axisymmetric Toroidal Plasmas," abstract 3B20 in Conference Proceedings.

- 5th Workshop on Fast Ignition Fusion Target, Madeira, Portugal, 18 - 22 June 2001.

1. V. Petržílka, L. Krlín and J.A. Tataronis, "Enhancement of Electron Acceleration in Plasma Beat-Wave Accelerators by an Additional Laser Beam," paper published in Proceedings of the 28th EPS Conference on Controlled Fusion and Plasma Physics.

- 43nd Annual Meeting of the Plasma Physics Division of the American Physical Society, Long Beach, CA, 29 October - 2 Novemebr 2001.

1. J.A. Tataronis and V. Petržílka, "Effects of the Injection Energy on Electron Acceleration in a Novel PBWA Configuration" - abstract published in Bull. Am. Phys. Soc. 46, 90 (2001).

2. J.A. Tataronis and A.H. Glasser, "The Ideal MHD Continuum in Nonaxisymmetric Toroidal Geometry" - abstract published in Bull. Am. Phys. Soc. 46, 166 (2001).

$-2002$

- 29th European Physical Society Conference on Controlled Fusion and Plasma Physics, Montreux, Switzerland 17 - 21 June 2002.

V. Petržílka, L. Krlín and J.A. Tataronis, "Electron Acceleration in a Plane Laser Beam Arising from Randomizing the Electron Motion by an Additional Laser Beam" -paper published in Conference Proceedings.

- 20th Symposium on Plasma Physics and Technology, Prague, Czech Republic, 10 - 13 June 2002.

1. V. Petržílka, L. Krlín and J.A. Tataronis, "Electron Acceleration in a Plane Laser Beam," paper published in Czech. J. Phys. 52 , Suppl. 3, p. 279 (2002). 
- 44th Annual Meeting of the Plasma Physics Division of the American Physical Society, Orlando, FL, 11 - 15 Novemebr 2002.

1. J.A. Tataronis, V. Petržilka and L. Krlín, "Controlled Electron Acceleration in a Plane Laser Beam" - abstract published in Bull. Am. Phys. Soc. 47, 335 (2002).

$-2003-$

- 30th European Physical Society Conference on Controlled Fusion and Plasma Physics, St. Petersburg, Russia, 7 - 11 July 2003.

1. V. Petržílka, L. Krlín and J.A. Tataronis, "Plasma Flows, Density Perturbations and Charge Separation Fields Resulting from Electron Acceleration in Two Crossed Laser Beams" - paper accepted for meeting presentation. 


\section{References}

[1] R. Kaiser and A. Salat, Phys. Rev. Lett. 77, 3133 (1996).

[2] Y. Pao, Nucl. Fusion 15, 631 (1975).

[3] J. A. Tátaronis, J. N. Talmadge, and J. L. Shohet, Comments Plasma Phys. Controlled Fusion 7, 29 (1982).

[4] E. Hameiri, Phys. Fluids 24, 562 (1981).

[5] E. Hameiri, Commun. Pure Appl. Math. XXXVIII, 43 (1985).

[6] J. P. Goedbloed, Phys. Fluids 18, 1258 (1975).

[7] A. H. Boozer, Phys. Fluids 24, 1999 (1981).

[8] J. A. Tataronis, V. Petržílka, V. Fuchs, and L. Krlín: Sherwood'97 Conf., paper $1 \mathrm{C} 28$.

[9] R.W. Motley et al., Phys. Rev. Letts. 43 (1979) 1799.

[10] V. Petržílka, F. Leuterer, F. Soeldner et al.: Nuclear Fusion 31 (1991) 1758.

[11] Y. Demers, private communication (1998).

[12] V. Petržílka and J. A. Tataronis: Bull. Am. Phys. Soc 42 (No. 10) 1930.

[13] B. D. Blackwell et al.: H-1 Project, Int. Stellarator Workshop, Madrid 1995, p. 26.

[14] B. Fischer, M. Krämer and Th. Enk: Plasma Phys. Controlled Fusion 36 (1994) 2003.

[15] S. Rauf and J.A. Tataronis, Phys. Rev. E 52, 4311 (1995).

[16] J.A. Tataronis and V. Petržílka, Phys. Plasmas 3, 4434 (1996).

[17] A. Pukhov and J. Meyer-ter-Vehn, in Superstrong Field in Plasmas, AIP Conf. Proc. 426 , p. 93.

[18] J. Meyer-ter-Vehn and Z. M. Sheng, Phys. Plasmas 6 (1999) 641.

[19] J.A. Tataronis and V. Petržílka, Bull. Am. Phys. Soc. 44 (1998). 